\title{
Online formative tests as a learning tool in engineering mathematics
}

\author{
$\begin{array}{ll}\text { P. Tobin } & \\ & \text { C. Lozanovski }\end{array}$
}

(Received 30 July 2007; revised 16 January 2008)

\begin{abstract}
We report on the results of the online mathematics project developed and implemented at Swinburne University of Technology. The trial was incorporated within the existing first year engineering mathematics course over the second semester of 2007. A detailed discussion is given on the structure of the item bank of mathematical multiplechoice questions and a brief description on the method by which they were generated. Over the duration of the trial, statistical data on several variables, including the distribution of incorrect student answers for a given multiple-choice question was collated. An example of the statistics of student responses based on Blackboard's tracking facility for one of the tests is presented. Finally, we discuss the future direction of the project.
\end{abstract}

See http://anziamj.austms.org.au/ojs/index.php/ANZIAMJ/article/view/343 for this article, (C) Austral. Mathematical Soc. 2008. Published January 22, 2008. ISSN 1446-8735 


\section{Contents}

1 Introduction

C377

2 Structure of online item bank: mathematical MCQS

C378

2.1 Method of generating mathematical MCQS . . . . . . . C C $\quad$ C378

2.2 Item bank of mathematical MCQs . . . . . . . . . . C379

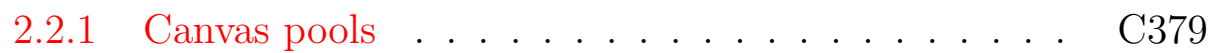

2.2.2 Randomly generated quizzes . . . . . . . . . . C380

2.2.3 Distracters . . . . . . . . . . . . . . . . . C381

2.2.4 Feedback to incorrect answers . . . . . . . . . . C382

3 Blackboard based statistics and student survey

C384

4 Discussion

C385

References

C389

\section{Introduction}

There is an ever increasing demand for online education in Australian Universities. The source of this demand comes not only from students who appear to eagerly embrace online active learning $[1,2,3,4,5,6,7]$ but also from Universities wishing to develop in-house educational software incorporated within the local learning management system (LMS). Online programs need not entirely replace traditional lectures. Web based materials such as e-quizzes and e-assignments can be adapted within a traditional (non-web based) course.

The aim of the trial at Swinburne University of Technology is to adapt an in-house developed web based item bank within existing course: Engineering Mathematics 1 and 2. The item bank comprises multiple canvas 
pools of mathematical multiple choice questions (MCQs) within the Blackboard environment. The existing LMS implemented by Swinburne University of Technology (Higher Education) is Blackboard.

The notes for these subjects are undergoing substantial change at present. The transition phase has seen the students referred to the existing university notes, the new online electronic notes and other online resources such as Wolfram online materials. If a student makes a mistake on a question, then they are referred to an appropriate section of one or all of these resources.

The motivation for this online formative testing is to provide additional assistance on demand for all engineering mathematics students but particularly those of weaker mathematical background including those articulating into the university from TAFE. The ready availability of the standard university web for each subject makes it an ideal tool. This extends the use of the web as a subject information resource in a natural way.

\section{Structure of online item bank: mathematical MCQS}

\subsection{Method of generating mathematical MCQS}

A straightforward method of creating mathematical MCQs in the Blackboard environment is to use the Equation Editor within Blackboard itself. However, an attempt to generate MCQs by this method proved inefficient; it is time consuming and laborious. An alternative, Respondus [8] (the most appropriate third party software required to upload canvas pools of MCQs) could also be used as its Equation Editor (or MathType) is more appropriate for the generation of mathematical questions. However, these methods of addressing the problem of generating MCQs are by no means the most efficient as each question requires manual input. 


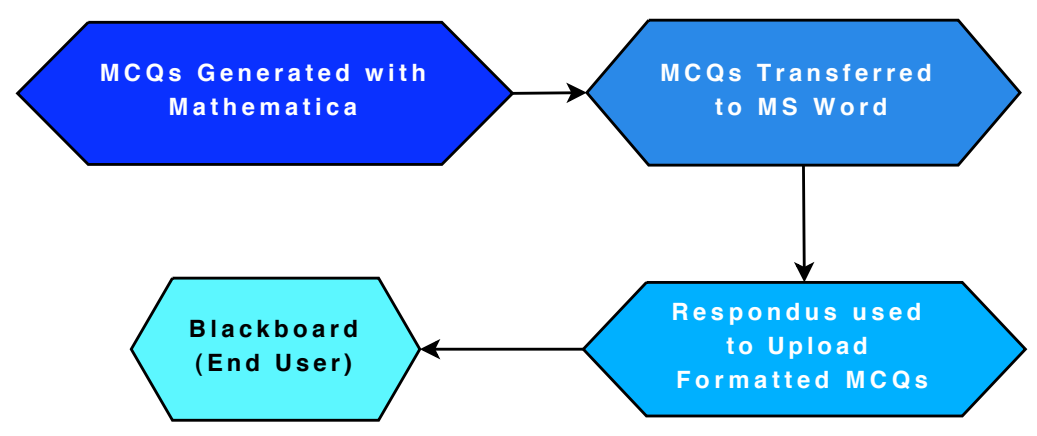

FiguRE 1: Diagram of the process of generating mathematical MCQs using four software packages; Mathematica, Microsoft Word, Respondus and the Learning Management system, Blackboard.

In comparison, an efficient method proposed in this study makes use of Mathematica, in conjunction with Microsoft Word (see Figure 1), to generate any number of 'similar' mathematical MCQs from a single Mathematica worksheet in a format dictated by Respondus. Although each Mathematica program must be written manually, there are clear advantages to this approach.

Similar use of Maple would have been an option. Mathematica was used as a license agreement existed with that company.

\subsection{Item bank of mathematical mcqs}

\subsubsection{Canvas pools}

For this trial there were 77 canvas pools each consisting of 100 'similar' mathematical MCQ. These questions have been sampled from the first year

${ }^{1}$ The meaning of 'similar' in this context is illustrated in Section 2.2.2. 
Engineering Mathematics course notes (HMS111 [9] and HMS112 [10]) and associated homework questions.

The questions in the canvas pool include the following topics: Graphics Calculator exercises, Algebra, Numbers, Functions, Linear algebra, Discrete Mathematics, Vectors, Calculus and Statistics. Moreover, the mathematical questions that have been implemented within the Blackboard environment include all the standard mathematical representations: graphics (dynamically generated by Mathematica), (static) diagrams, numerical and symbolic questions and answers.

\subsubsection{Randomly generated quizzes}

Over the duration of the trial, a number of online math quizzes were set each consisting of ten questions. Students have the option of repeating the quiz as many times as required. The quizzes were generated by randomly sampling from each of the canvas pools (Level 1 randomisation) chosen for that particular quiz. For example, the topics covered from the first quiz are 'TI-83 Calculator exercises' and 'Algebra'. Each canvas pool contains $N$ 'similar' questions (currently $N=100$ ), that is the underlying mathematical method to solve the problem is the same although no two questions are exactly the same in form. To illustrate this point consider the following. We aim to test the student with a simple quadratic equation. Suppose solving the following quadratic equation

$$
x^{2}+x+1=0,
$$

is listed as the first question in its canvas pool. Then the $k$ th $(1 \neq k \in N)$ question could be, for example,

$$
x^{2}+x+3=0,
$$

or 'similar' but not (1). No two questions in a canvas pool are equivalent. 
This Level 2 randomisation ensures that there will be a high probability that no two students will receive identical quizzes. For this trial, there are 77 canvas pools of one hundred questions making up the first year Engineering Mathematics item bank in the two subjects Engineering Mathematics 1 and 2 .

\subsubsection{Distracters}

For any given mathematical MCQ in the canvas pool, the number of distracters is set to randomly vary, three to six in number, while the position of the correct answer is also randomly chosen. Note that the number of distracters can be fixed for any given test; this was required for the trial. For this trial the number is standardised to four distracters. The distracters are algorithmically calculated as perturbations from the correct answer and thereby appear as plausible alternatives. However, it is more desirable to generated distracters that have been gathered from common student mistakes. Perhaps a combination of both would be ideal.

How are the distracters generated? Suppose the solution of a mathematical problem is $x=1.23$. Mathematica would then generate the distracters by first calculating a random set of positive, no repeating, integers on an appropriate interval, for example $\{5,8,6,9\}$. Each distracter, uniquely corresponding to an element of this set, is generated by adding \pm the reciprocal of each element to the correct answer. Thus, an associated set of distracters could be $\{1.43,1.06,1.11,1.34\}$. Note that if any distracters are equal after rounding-off then the program discards that set of distracters and regenerates another. However, in some cases there is no particular need to resort to decimals as the same approach can be used to generate the following example.

Example 1 Consider the linear system of equations

$$
\left(\begin{array}{cc}
1 & 3 \\
2 & -3
\end{array}\right)\left(\begin{array}{l}
x \\
y
\end{array}\right)=\left(\begin{array}{l}
0 \\
5
\end{array}\right),
$$


with solution

$$
(x, y)=\left(\frac{5}{3},-\frac{5}{9}\right),
$$

where the set of Mathematica generated distracters may, for example, appear as

$$
\begin{aligned}
& (x, y)=\left(\frac{-5}{3}, \frac{5}{9}\right), \\
& (x, y)=\left(\frac{7}{3},-\frac{5}{9}\right), \\
& (x, y)=\left(\frac{5}{9},-\frac{5}{3}\right), \\
& (x, y)=\left(\frac{4}{3},-\frac{5}{9}\right) \\
& (x, y)=\left(\frac{5}{3},-\frac{4}{9}\right) .
\end{aligned}
$$

The corresponding question for Example 1 on the Blackboard environment would appears as in Figure 2.

\subsubsection{Feedback to incorrect answers}

An important facility within the Blackboard environment is the feedback option for incorrect student answers to quiz questions, see Figure 3. This option displays the overall score and, the incorrect and correct answers. In addition, it facilitates an external hyper-link - in this trial external hyperlinks are attached to appropriate course materials: (sub/subsub-)sections or examples. The student has an opportunity to review the relevant course material online and the option of re-sitting the quiz. Note that if a student decides to re-sit the quiz then each quiz question will, most likely, not be exactly the same as in the previous quiz (Level 2 randomisation). 
The solution for the linear system of equations

$$
\left(\begin{array}{cc}
1 & 3 \\
2 & -3
\end{array}\right)\left(\begin{array}{l}
x \\
y
\end{array}\right)=\left(\begin{array}{l}
0 \\
5
\end{array}\right)
$$

is $(x, y)=$

$$
\begin{array}{cc}
\text { C } & (-5 / 3,5 / 9) \\
\text { C } & (7 / 3,-5 / 9) \\
\text { c } & (5 / 3,-5 / 9) \\
\text { C } & (5 / 9,-5 / 3) \\
\text { C } & (4 / 3,-5 / 9) \\
\text { C } & (5 / 3,4 / 9)
\end{array}
$$

FigurE 2: Example of Mathematica generated MCQ with distracters as it appears in Blackboard. 


\section{Question 1}

The solution for the linear system of equations

$$
\left(\begin{array}{ll}
1 & 3 \\
2 & -3
\end{array}\right)\left(\begin{array}{l}
x \\
y
\end{array}\right)=\left(\begin{array}{l}
0 \\
5
\end{array}\right)
$$

is $(x, y)=$

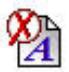

Selected Answer: $\times(7 / 3,-5 / 9)$

Correct Answer: $\checkmark(-5 / 3,5 / 9)$

Feedback: You have selected an Incorrect Answer. Please review the following section(s) of your notes and try again: Click Here In addition, you can also see Wolfram MathWorld

FiguRE 3: Example of feedback to incorrect student answer in Blackboard.

On the other hand, the feedback facility itself can be used to gather data on students' incorrect answers. A required resource to be acquired from this trial is a database of students' incorrect answers in order to generate more enticing distracters on a large scale.

\section{Blackboard based statistics and student survey}

Blackboard has very simple statistical output on the quizzes. It will provide charts on student attempts broken down by student (see Figure 4) and by question (see Figure 5). It will differentiate the item success. However, as students all receive different variants of the same question it is only a partially suitable comparison. The important output is seeing the student 


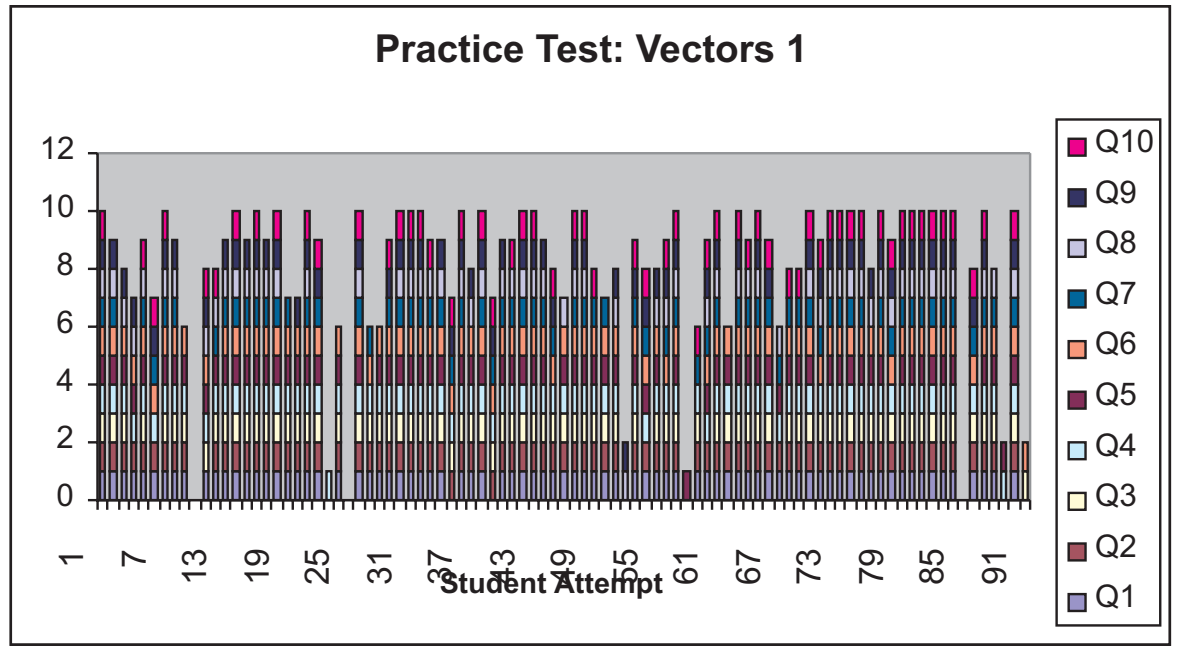

Figure 4: Plot of student attempts against correct answers for the test on vectors.

attempts are occurring and noting that broadly the earlier questions give more successful responses than the later ones. This suggests that the degree of difficulty progresses with item number in the tests.

Blackboard also issues simple statistics such as numbers of attempts, correct answer numbers and similar data with means and variances as well as maxima and minima.

\section{Discussion}

This program, as report here, is the first step towards fulfilling the aim of providing a teaching and learning tool for engineering mathematics students. Currently, the distracters are generated as perturbations from the correct answer. The next step involves improving the tests by constructing databases 


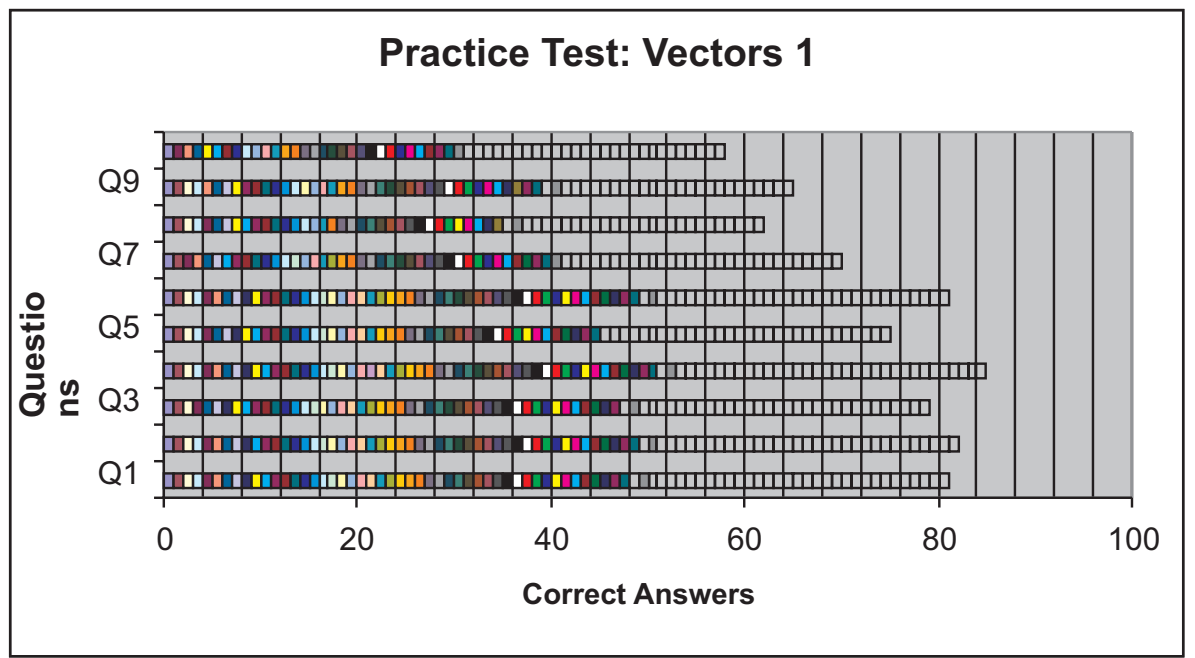

FiguRE 5: Plot of correct answers for a given question in the vector test canvas pool. Note that colours here merely distinguish answers given for a question. 


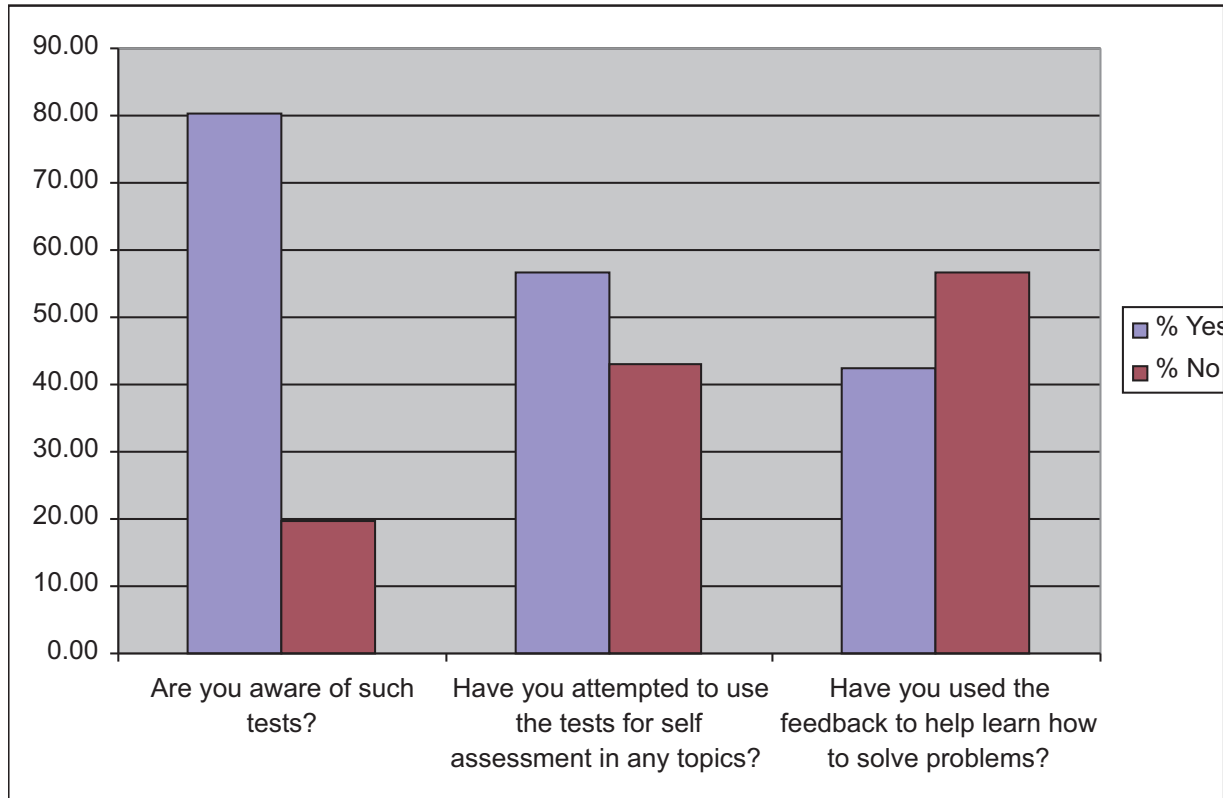

FIGURE 6: Results of student survey conducted in semester 2. These results indicate that future advertising of the program should also focus on the formative feedback facility.

of pre-determined distracters, constructed from common student mistakes. This step is essential as students could learn why they made a mistake by way of the formative feedback facility which could specifically address a range of possible errors commonly encountered.

In order to assess the feasibility of the program a student survey was conducted in semester 2, subjects HMs112 (engineering mathematics) and HMS112P (engineering mathematics 2, product design). A total of 132 students responded with the results displayed in Figures 6 and 7 . The task was to determine, in the first place, if the students were aware of this new learning tool and if they actually used it during the course of their studies in 


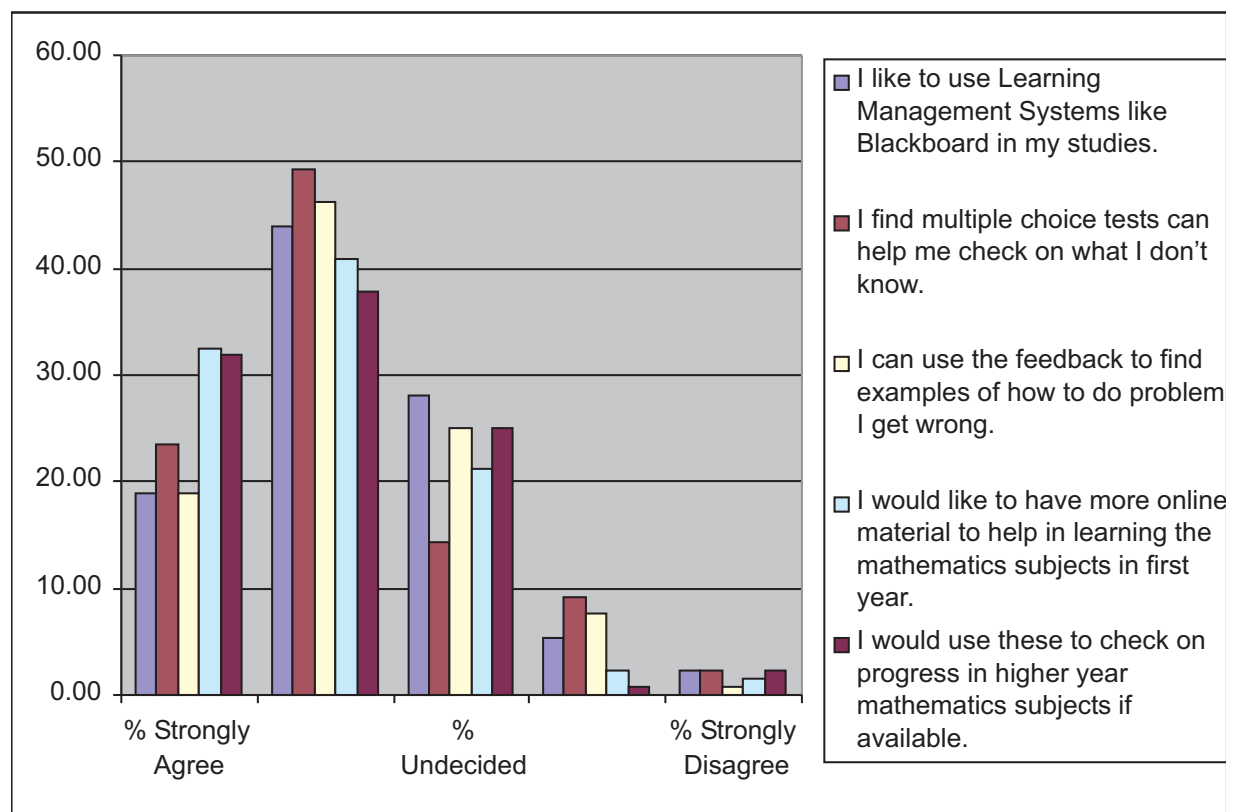

Figure 7: Results of student survey conducted in semester 2. Clearly, most student agree with all the question posed. 
semester 2. It is encouraging to note that the majority of students express a need for online support programs, like the one under development here, perhaps this indicates a desire to rapidly embrace new learning technologies. Moreover, there appears to be overwhelming support for mathematical multiple choice questions as a way of testing students knowledge.

The long term objective of the program is to increase performance by the students and in particular to improve the weaker tail of students. This may take some time to determine and will be among our aims for future development of the program.

Acknowledgements: The authors are grateful to the anonymous referees for constructive comments on a previous draft of this paper. We acknowledge the assistance of the university's Academic Development and Support unit in a pilot program and in their assistance on technical aspects of the present project. We also acknowledge discussions held with Jim McGovern of RMIT on the early plan for the project. Finally the Learning and Teaching Performance Fund at Swinburne is funding the development of much of the program through the Faculty of Engineering and Industrial Sciences and their assistance is gratefully acknowledged.

\section{References}

[1] M. Boylan, What have we learned from 15 years of supporting the development of innovative teaching technology?, Social Science Computer Review, vol. 22, no. 4, p. 405, 2004. doi:10.1177/0894439304268646 C377

[2] A. Zemel, F. Xhafa, and G. Stahl, Analyzing the organization of collaborative math problem-solving in online chats using statistics and conversation analysis., Lecture notes in computer science, no. 3706, 
p. 271, 2005. http://www. springerlink. com/content/ d4h9fyp05hnp052d/fulltext.pdf C377

[3] Q. Li, Would we teach without technology? a professor's experience of teaching mathematics education incorporating the internet., Educational Research, vol. 45, no. 1, p. 61, 2003. doi:10.1080/0013188032000086127 C377

[4] D. Bourne, A self-paced course in pharmaceutical mathematics using web-based databases., American Journal of Pharmaceutical Education, vol. 5, no. 70, p. 271, 2006. http://www.pubmedcentral.nih.gov/ picrender.fcgi?artid=1637015\&blobtype=pdf C377

[5] W. Hwang, N. Chen, and R. Hsu, Development and evaluation of multimedia whiteboard system for improving mathematical problem solving., Computers and Education, vol. 46, no. 2, p. 105, 2006. doi:10.1016/j.compedu.2004.05.005 C377

[6] Q. Li, Infusing technology into a mathematics methods course: any impact?, Educational Research, vol. 47, no. 2, p. 217, 2005. doi:10.1080/00131880500104341 C377

[7] G. Stefansson, The tutor-web: An educational system for classroom presentation, evaluation and self-study., Computers and Education, vol. 43, no. 4, p. 315, 2004. doi:10.1016/j.compedu.2003.09.004 C377

[8] Respondus version 3.5: http://www.respondus.com, Copyright 2000-2007 Respondus, Inc., 2007. C378

[9] C. Barling, N. Li, D. Lucy, D. Richards, J. Sampson, J. Steiner, and P. Tobin, HMS111: Engineering Mathematics 1, Copyright 2006 Swinburne University of Technology, 2007. C380

[10] B. Leary, D. Lucy, J. Sampson, D. Gilliam, and D. Roberts, HMS112: Engineering Mathematics 2, Copyright 2006 Swinburne University of Technology, 2006. C380 


\section{Author addresses}

1. P. Tobin, Mathematics, FEIS, Swinburne University of Technology, Hawthorn, Australia.

2. C. Lozanovski, Mathematics, FEIS, Swinburne University of Technology, Hawthorn, Australia. mailto:clozanovski@swin.edu.au 